\title{
COSMOGENIC TAU NEUTRINO INDUCED RADIO EMISSION
}

\author{
KWANG-CHANG LAI \\ Institute of Physics, National Chiao Tung University, Hsinchu, Taiwan \\ Leung Center for Cosmology and Particle Astrophysics, National Taiwan University \\ Taipei, Taiwan \\ kclai@mail.nctu.edu.tw \\ GUEY-LIN LIN \\ Institute of Physics, National Chiao Tung University, Hsinchu, Taiwan \\ Leung Center for Cosmology and Particle Astrophysics, National Taiwan University \\ Taipei, Taiwan \\ TSUNG-CHE LIU \\ Institute of Physics, National Chiao Tung University, Hsinchu, Taiwan \\ JIWOO NAM \\ Department of Physics, Ewha Womans University, Seoul, Korea \\ CHI-CHIN CHEN \\ Institute of Astrophysics, National Taiwan University, Taipei, Taiwan
}

\begin{abstract}
Cosmogenic neutrinos ${ }^{1}$ are expected from ultrahigh energy cosmic rays undergoing the GZK process ${ }^{2,3}$ and anticipated to be observed by detection of the air showers from the decays of tau leptons. We use CORSIKA simulated shower structure to Calculate the coherent geosynchrotron radio emissions of the tau decay showers above $10^{17} \mathrm{eV}$. We present the pattern and spectrum of the radio waves and discuss their detections by radio antennae.
\end{abstract}

\section{Introduction}

The origins of the ultra-high energy cosmic rays remain a fundamental and unsolved problem in astroparticle physics. Promising clues could be provided by the associated high energy neutrinos since they would neither interact with intergalactic or interstellar media nor be deflected by the magnetic fields. Various detectors have been proposed for detecting high energy neutrinos. Some of them rely on measuring the air shower by the so-called earth-skimming $\nu_{\tau}$, for which horizontal showers are generated by the ensuing $\tau$ decay. ${ }^{4}$ The fluorescence light and Cherenkov radiation generated by the air shower have been extensively studied and adapted in many projects. In this paper, we calculate the geosynchrotron radiation from the 
$\nu_{\tau}$ induced air showers since this detection method is quite promising. The strongly directional radio antennae are simple to build.

In Sec. 2, we briefly discuss the coherent geosynchrotron emission scenario initiated in 1970's ${ }^{5}$ and further developed by Huege and Falcke. ${ }^{6}$ Unlike their analytical parametrization for shower profile, we provide the CORSIKA ${ }^{7}$ simulated shower profile in Sec. 3 for further calculation of geosynchrotron radiation in Sec. 4. In Sec. 5 , we summarize and conclude our work.

\section{Coherent Geosynchrotron Emission}

Radio emissions from the shower are produced via two mechanisms: Cerenkov radiation from charge excess moving with a velocity higher than the speed of light in the traversed medium (the so-called Askaryan effect ${ }^{8}$ ) and acceleration of charged particles in the earth's magnetic field. ${ }^{6}$ In this work, we investigate the synchrotron radiation from the latter in the scenario developed by Ref. 6 .

This model is based on the synchrotron theory discussed in the standard text. ${ }^{9}$ In far-field limit (distance $R$ to the observer larger than the extent of the particle trajectory), the measure of the frequency $\omega$ of the electric field, $A(R, \omega)$, can be divided into two orthogonal components $A_{\perp}$ and $A_{\|}$such that

$$
A(R, \omega)=\frac{\omega e}{\sqrt{8 c} \pi} \exp \left\{i\left(\omega \frac{R}{c}-\frac{\pi}{2}\right)\right\}\left[-\hat{e}_{\|} A_{\|}(\omega) \pm \hat{e}_{\perp} A_{\perp}(\omega)\right],
$$

where the plus and minus signs are used for electrons and positrons respectively, with $e$ denoting the unit charge and the unit vectors $\hat{e}_{\|}$and $\hat{e}_{\perp}$ defined as in Ref. $6 .{ }^{\mathrm{a}}$ The two components are given by

$$
\begin{aligned}
& A_{\|}(\omega)=i \frac{2 \rho}{\sqrt{3} c}\left(\frac{1}{\gamma^{2}}+\theta^{2}\right) K_{2 / 3}(\xi), \\
& A_{\perp}(\omega)=\theta \frac{2 \rho}{\sqrt{3} c}\left(\frac{1}{\gamma^{2}}+\theta^{2}\right)^{1 / 2} K_{1 / 3}(\xi),
\end{aligned}
$$

with

$$
\xi=\frac{\omega \rho}{3 c}\left(\frac{1}{\gamma^{2}}+\theta^{2}\right)
$$

where $K_{a}$ denotes the modified Bessel function of order $a, \gamma$ denotes the Lorentz factor of the charged particle, $\theta$ denotes the angle between the line-of-sight and the tangent of the particle trajectory. The curvature radius $\rho$ of the instantaneous particle trajectory is given by

$$
\rho=\frac{v \gamma m_{e} c}{e B \sin \alpha}
$$

where $B$ is the magnetic field strength and $\alpha$ is the pitch angle between the particle trajectory and magnetic field direction.

athe detailed geometry is given in Ref. 6. 
In the air shower, electrons and positrons are created in pairs. In this model, each electron-positron pair is assumed having perfectly symmetric trajectories such that $A_{\|}$components from the two particles add up to $2 A_{\|}$, whereas $A_{\perp}$ contributions completely cancel each other. This is, however, too simplified and does not adequately represent the realistic case. Due to the variation of the directions from which the observer sees the particle pair, the loss of coherence depends on the misalignment between the electron and the positron in a pair. To assure the validity of coherent addition of the positron and electron emission, a particle density over $1000 \mathrm{~m}^{-3}$ is required in the shower front. We choose the particles' instantaneous velocity vectors to point out radially away from the spherical surface of the shower front. Thus, the symmetric trajectory is proved.

In this scenario, the emission from an "effective" electron-positron pair can be approximated as that from a perfect pair, with only $A_{\|}$remaining. Therefore, we can drop the difference between positrons and electrons and the spectrum emitted by such a pair is

$$
E(R, \omega)=\left(\frac{4 \pi}{c}\right)^{1 / 2} \frac{1}{R} \frac{2 \omega e}{\sqrt{8 c} \pi} e^{i\left(\frac{\omega R}{c}-\frac{\pi}{2}\right)}\left(-\hat{e}_{\|}\right) A_{\|}(\omega) .
$$

Given the particle distribution in the shower front, superposition renders the collective spectrum of the radio emission.

\section{Air Shower Simulations}

The tau decay induced air shower is initiated by the decay product and the dominant channels are listed in Table 1:

\begin{tabular}{lc}
\hline channel & Branching ratio(\%) \\
\hline$e^{-} \bar{\nu}_{e} \nu_{\tau}$ & 18 \\
$\mu^{-} \bar{\nu}_{\mu} \nu_{\tau}$ & 17 \\
$\pi^{-} \nu_{\tau}$ & 11 \\
$\pi^{-} \pi^{0} \nu_{\tau}$ & 25 \\
\hline
\end{tabular}

Only four main channels are listed while others are ignored since they either have too small branching ratios or have more than 3 decay products. For an Earthskimming tau lepton with an energy of $10^{17} \mathrm{eV}$, the tau decay length is about $5 \mathrm{~km}$ and the resulting shower reaches its maximum at $10 \mathrm{~km}$.

Using CORSIKA code, we simulate the shower initiated by electrons at five different energies. Simulation shows that the shower particles reside in a shower thickness less than $1 \mathrm{~m}$. Compared with the radiation traversing distances of $\sim 10$ $\mathrm{km}$, the shower front at the maximum is treated as longitudinally coherent. The 
remaining structures are lateral profile and Lorentz factor distribution representing the spatial and energy distribution of the shower particles.

\section{Radio Properties}

Having the spatial structure and energy distribution of shower particles determined, we can calculate the emission from the shower maximum. The phase differences between pulses from the individual particles lead to the spectrum significantly different from that due to fully coherent pulses. The spectrum of the radio emission is calculated, by superposition, as

$$
\begin{aligned}
E(\omega)= & \frac{1}{N} \int_{\gamma_{\min }}^{\gamma_{\max }} d \gamma \int_{0}^{2 \pi} d \varphi \int_{0}^{r_{M} / K} d \vartheta K^{2} \sin \vartheta \\
& \times p(\gamma) \rho(r(\vartheta, \varphi)) E(R(\vartheta, \varphi))
\end{aligned}
$$

where

$$
\begin{aligned}
N & =\int_{\gamma_{\min }}^{\gamma_{\max }} d \gamma p(\gamma), \\
& \simeq \int_{0}^{2 \pi} d \varphi \int_{0}^{r_{M} / K} d \vartheta K^{2} \sin \vartheta \rho(r(\vartheta, \varphi)) .
\end{aligned}
$$

with $r_{\mathrm{M}}$ and $K$ denoting the Moliere radius and the curvature radius of the shower front at its maximum. $p(\gamma)$ and $\rho(r(\vartheta, \varphi))$ are the energy distribution in terms of Lorentz factor $\gamma$ and lateral distribution in the shower front at position $r(\vartheta, \varphi)$ of the charged particles. $K=1000 \mathrm{~m}$ is deduced from the simulation. We set $r_{\mathrm{M}}=100$

$\mathrm{m}$ and $\left(\gamma_{\min }, \gamma_{\max }\right)=(1,1000)$, for most particles are taken into account.

In Fig. 1, we plot the spectrum received in the center of the area illuminated by the shower maximum for different shower energies. The radio strength scales as the shower energy and, hence, the charged particles accounted. Fig. 2 demonstrates the off-set dependence electric field.

In Fig. 3, we calculate the pulse measured by a receiver with a given bandwidth. Although the pulse amplitude drops noticeably as the observer moves away from the center of the illuminated area to far distances, its strength is still large enough to be detected as far as $1000 \mathrm{~m}$ in the lateral direction.

\section{Discussion and Conclusion}

We analyze properties of coherent geosynchrotron radio emission from the earthskimming tau neutrino induced air shower. We use CORSIKA simulated shower structure for a more realistic calculation of the field strength of the radio emission. Assuming three logarithmic-periodic dipole antennae phase coupled as described in Ref. 10, the measurement of a $10^{17} \mathrm{eV}$ shower would be feasible up to an offset distance of $\sim 1500 \mathrm{~m}$ which is much larger than $300 \mathrm{~m}$ obtained in Ref. 10 with a $10^{16} \mathrm{eV}$ shower energy. For the integrated cosmogenic tau neutrino flux ${ }^{11,12}$ 




Fig. 1. (Color online) Frequency dependence of $|E(R, 2 \pi 100 \mathrm{MHz})|$ for the maximum at the observation distance of $10 \mathrm{~km}$. Curves in blue, red, yellow and green represent $10^{17} \mathrm{eV}, 10^{17.5} \mathrm{eV}, 10^{18} \mathrm{eV}$ and $10^{18.5} \mathrm{eV}$ showers, respectively.

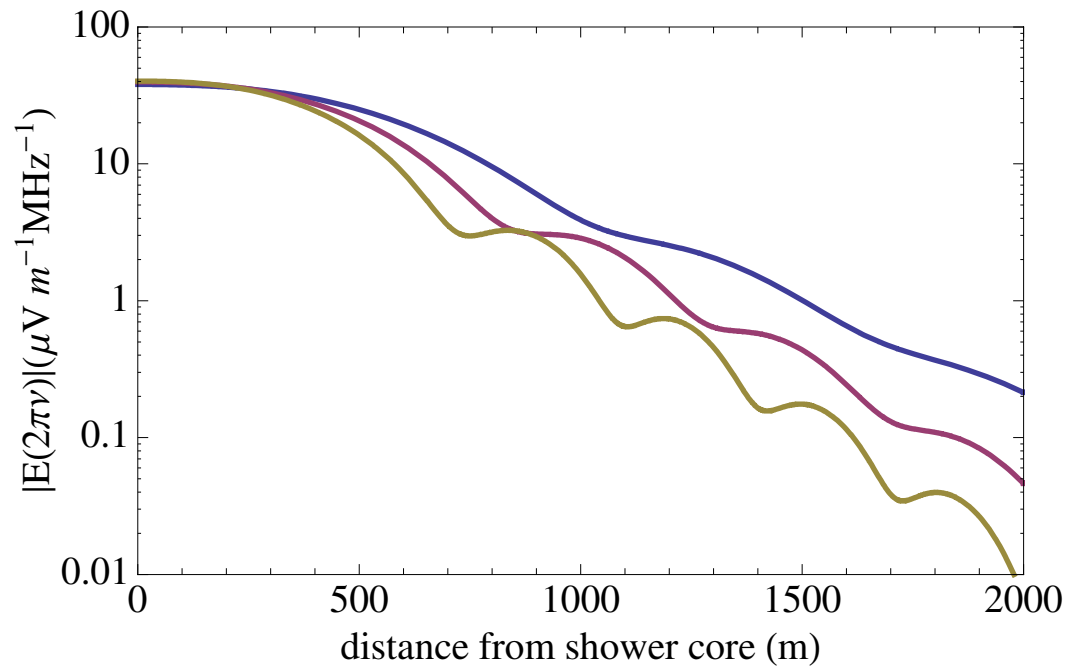

Fig. 2. (Color online) Off-set dependence of $|E(R, 2 \pi \times \nu)|$ for the maximum of a $10^{17} \mathrm{eV}$ shower at the observation distance of $10 \mathrm{~km}$. Curves in blue, red and yellow represent signals in observing frequencies of $50 \mathrm{MHz}, 75 \mathrm{MHz}$ and $100 \mathrm{MHz}$, respectively.

$\phi_{\nu_{\tau}} \sim 10^{-17} \mathrm{~cm}^{-2} \mathrm{~s}^{-1} \mathrm{sr}^{-1}$ above $10^{18} \mathrm{eV}$, the event rate of tau neutrino observation in our estimation will therefore be $\sim 0.08$ per year.

So far we only calculate the radio strength approximatively and conservatively. To refine our work, we shall carry a more realistic and sophisticated calculation 


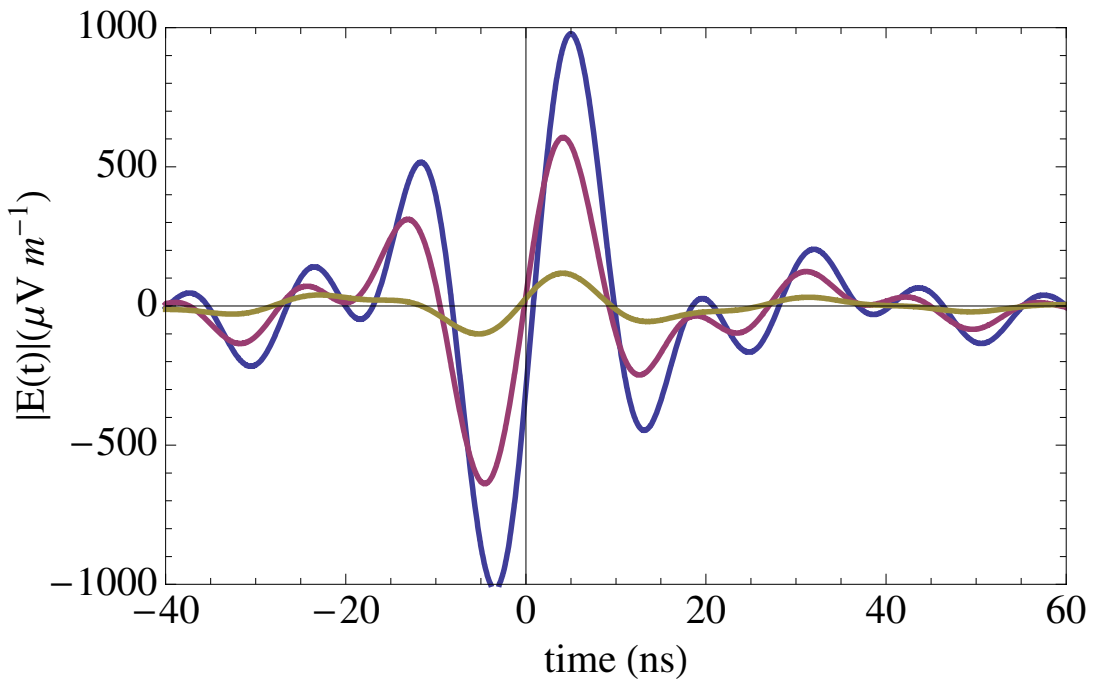

Fig. 3. (Color online) Reconstructed pulses from emission of a $10^{17} \mathrm{eV}$ shower at the observation distance of $10 \mathrm{~km}$, using an idealized rectangular filter spanning $30-80 \mathrm{MHz}$. Curves in blue, red and yellow denote pulses measured at center, at lateral distances of 500m and 1000m, respectively.

which takes into account in detail the conversion from tau neutrinos to tau leptons and the detection efficiency of the designed antennae.

\section{Acknowledgments}

KCL and GLL are supported by the National Science Council (NSC 097-2811-M009-029 and 096-2112-M-009-023-MY3, respecttively) of Taiwan.

\section{References}

1. V. S. Berezinsky and G. T. Zatsepin, Phys. Lett. 28B, 423(1996).

2. K. Greisen, Phys. Rev. Lett. 16, 748(1966).

3. G. T. Zatsepin and V. A. Kuzmin, Sov. Phys. JETP Lett. 4, 78(1966).

4. D. Fargion, Astrophys. J. 570, 909(2002).

5. H. R. Allan, Prog. in Element. Part. and Cos. Ray Phys. 10, 171(1971).

6. T. Huege and H. Falcke, A\& A 412, 19-34 (2003).

7. D. Heck, J. Knapp, J. N. Capdevielle, G. Schatz and T. Thouw, FZKA Report 6019, Forschungszentrum Karlsruhe, 1998.

8. G. A. Askaryan, Sov. Phys. JETP 14, 441(1962) and 21, 658(1965).

9. J. D. Jackson, Classical Electrodynamics, 2nd ed. (New York: John Wiley \& Sons, 1975).

10. O. Brusova, L. Anchordoqui, T. Huege and K. Martens, 30th International Cosmic Ray Conference, arXiv:0708.3824[astro-ph].

11. R. Engel, D. Seckel and T. Stanev, Phys. Rev. D 64, 093010(2001).

12. J.-J. Tseng, T.-W. Yeh, H. Athar, M. A. Huang, F.-F. Lee and G.-L. Lin, Phys. Rev. D 68, 063003 (2003). 